\title{
p53-independent mechanisms regulate the P2-MDM2 promoter in adult astrocytic tumours
}

\author{
M Dimitriadi*,', G Poulogiannis', L Liu', LM Bäcklund², DM Pearson', K Ichimura' and VP Collins' \\ 'Division of Molecular Histopathology, Department of Pathology, University of Cambridge, Level 3, Lab Block, Addenbrooke's Hospital, Box 231 , \\ Cambridge CB2 0QQ, UK; '2 Department of Oncology-Pathology, Karolinska University Hospital, Stockholm 17I 76, Sweden
}

The MDM2 gene is amplified and/or overexpressed in about $10 \%$ of glioblastomas and constitutes one of a number of ways the p53 pathway is disrupted in these tumours. MDM2 encodes a nuclear phosphoprotein that regulates several cell proteins by binding and/ or ubiquitinating them, with p53 being a well-established partner. MDM2 has two promoters, $\mathrm{PI}$ and $\mathrm{P} 2$ that give rise to transcripts with distinct $5^{\prime}$ untranslated regions. Transcription from P2 is believed to be controlled by $\mathrm{p} 53$ and a single-nucleotide polymorphism (SNP309, T > G) in P2 is reported to be associated with increased risk for, and early development of, malignancies. The use of PI and P2 has not been investigated in gliomas. We used RT-PCR to study PI - and P2-MDM2 transcript expression in astrocytic tumours, xenografts and cell lines with known MDM2, TP53 and p $14^{A R F}$ gene status. Both promoters were used in all genetic backgrounds including the use of the P2 promoter in TP53 null cells, indicating a p53-independent induction of transcription. Transcripts from the PI promoter formed a greater proportion of the total MDM2 transcripts in tumours with MDM2 amplification, despite these tumours having two wild-type TP53 alleles. Examination of SNP309 in glioblastoma patients showed a borderline association with survival but no apparent correlation with age at diagnosis nor with TP53 and p/4ARF status of their tumours. Our findings also indicate that elevated MDM2 mRNA levels in tumours with MDM2 amplification are preferentially driven by the PI promoter and that the P2 promoter is not only regulated by $\mathrm{p} 53$ but also by other transcription factor(s).

British Journal of Cancer (2008) 99, I | 44- I I52. doi: 10. I038/sj.bjc.6604643 www.bjcancer.com

Published online 9 September 2008

(c) 2008 Cancer Research UK

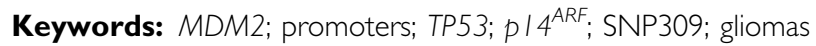

The evolutionarily conserved MDM2 gene (12q15) encodes a 491 amino-acid nuclear protein, whose activity and cellular localisation is believed to be controlled by post-translational modifications (Meek and Knippschild, 2003). For example, phosphorylation of MDM2 at Ser-166 and Ser-186 by the protein kinase Akt (also known as PKB) results in nuclear entry (Ashcroft et al, 2002). MDM2 is an E3 ubiquitin ligase, known to negatively regulate p53 by blocking its trans-activation domain and targeting it for ubiquitination and proteasome-mediated degradation (Momand et al, 1992; Haupt et al, 1997; Kubbutat et al, 1997). The tumour suppressor protein $\mathrm{p} 14^{\mathrm{ARF}}$ associates with and inhibits the E3 ubiquitin ligase activity of MDM2 permitting an accumulation of p53 and a consequent transcriptional response (Kamijo et al, 1998). MDM2 interacts with many other proteins including $\mathrm{Rb} 1, \mathrm{E} 2 \mathrm{~F} 1$, and the ribosomal proteins L5, L11 and L23, indicating that MDM2 is involved in a complex circuit of interactions, affecting among others the cell cycle and apoptosis (Zhang and Zhang, 2005). The details of the control of these interactions have still to be determined.

MDM2 is amplified and/or overexpressed in a variety of human tumours of diverse tissue origins (Momand et al, 1998). Up to $10 \%$ of the most malignant astrocytic tumours, glioblastomas (WHO malignancy grade IV) (Louis et al, 2007) show MDM2 gene

*Correspondence: Dr M Dimitriadi; E-mail: mdimitriadi@cantab.net Received 8 May 2008; revised 18 July 2008; accepted 6 August 2008; published online 9 September 2008 amplification with consequent mRNA overexpression. This is generally associated with primary (de novo) glioblastomas that have wild-type TP53 and p14 ${ }^{A R F}$ alleles (Reifenberger et al, 1993; Ichimura et al, 2000). Consequently, amplification and/or overexpression of $M D M 2$ is believed to be an alternative mechanism for escaping p53-regulated control (Ichimura et al, 2000).

$M D M 2$ gene transcription is regulated by two promoters, $\mathrm{P} 1$ and $\mathrm{P} 2$. The P1 promoter is located upstream of exon 1 and is active at basal constitutive levels in most cells (Mendrysa and Perry, 2000). Although motifs of the $\mathrm{P} 1$ promoter important for its activity have been defined, its control is still not understood (Chang et al, 2004; Phillips et al, 2006). The second promoter (P2) is located in intron 1 , it has two p53-responsive elements (Zauberman et al, 1995) and p53 is believed to initiate MDM2 transcription from this promoter, thus forming an auto-regulatory feedback loop. Other p53-independent mechanisms have also been proposed (Qi et al, 1999; Ries et al, 2000; Phelps et al, 2003, 2005). In addition, a T $>$ G polymorphism, referred to as SNP309 (rs2279744) in intron 1 of the human MDM2 gene, has been suggested to affect P2 activity by increasing the binding affinity of the Sp1 transcription factor (Bond et al, 2004). This is thought to result in higher MDM2 protein levels, which would potentially attenuate the p53 pathway and might facilitate cancer formation (Bond et al, 2004).

Transcripts derived from the $\mathrm{P} 1$ promoter (P1-MDM2) do not utilise exon 2 and have exon 1 as their $5^{\prime}$ untranslated region ( $5^{\prime}$ UTR), whereas P2-derived transcripts (P2-MDM2) have exon 2 (Barak et al, 1994). Bases 5-7 of exon 3 form the start codon and 
are common to transcripts from both promoters. Translation of transcripts from the $\mathrm{P} 1$ promoter is eight-fold less efficient than translation of the P2 promoter transcripts (Landers et al, 1997), probably due to the presence of two short upstream open reading frames located in exon 1 (Jin et al, 2003).

The majority of studies have examined total MDM2 mRNA expression in normal and tumour tissues (Bueso-Ramos et al, 1995; Broll et al, 1999; Ko et al, 2000; Miyajima et al, 2001). The individual activity of the P1 and P2 promoters has been investigated in breast and oral cancer and head and neck squamous cell carcinoma (Ralhan et al, 2000; Millon et al, 2001; Okumura et al, 2002), but not in human gliomas. We have assessed how the gene status of MDM2, TP53 and $p 14^{A R F}$ influences promoter usage in astrocytic gliomas (primary tumours, glioblastoma xenografts, glioblastoma cell lines). In addition, the SNP309 status was studied in glioblastoma patients and correlated to a number of genetic (i.e., TP53 and p14 ${ }^{A R F}$ ) and clinical (i.e., survival and age at diagnosis) parameters.

\section{MATERIALS AND METHODS}

\section{Tumour tissue, xenografts and cell lines}

A total of 73 gliomas including 56 glioblastomas (prefixed as GB), 10 anaplastic astrocytomas (prefixed as AA) and 7 astrocytomas (prefixed as A) were used in the study. In addition, xenografts from three glioblastomas and three glioma cell lines were studied. The primary tumours were classified according to WHO classification (Louis et al, 2007). Each tumour piece analysed had a minimum tumour cell content of $75 \%$ but generally greater than $90 \%$, as assessed by histology. The tumours have been included in previous studies using the same identification numbers (Reifenberger et al, 1993; Ichimura et al, 2000, 2008; Liu et al, 2005). Xenografts hold the same number as the tumour from which they were derived with the suffix $\mathrm{X}$ followed by passage number. Collection and handling of tumour tissues and xenografts were as described (Schmidt et al, 1999; Ichimura et al, 2000). The characteristics of the cell lines Tp365MG and Tp265MG have been reported elsewhere (Collins, 1983). CCF-STTG1 was purchased from ATCC (Middlesex, UK). Supplementary Table 1 lists specimen numbers and their diagnosis, and indicates in which part of the study each specimen was used. Table 1 lists the MDM2, TP53 and $p 14^{A R F}$ gene status of the tissues, xenografts and cell lines used. The study was approved by the Ethical Committee of the Karolinska Hospital (No. 91:16) and the Cambridge Local Research Ethics Committee, Cambridge, UK (ref. LREC 03/115).

\section{TP53 analysis by multiplex PCR and MDM2 SNP309 genotyping}

DNA extraction from patients' peripheral blood and cell lines was as described previously (Ichimura et al, 1996). Multiplex PCR was performed by amplifying exon 2 (PC1046/PC1047) and exon 5 (PC929/PC931) of the TP53 gene together with exon 35 (PC2419/ PC2420) of an internal control gene (DEPDC5). The latter is known to be genetically normal in the samples investigated (Seng et al, 2005). Products were carried out on $2 \%$ agarose gels and visualised by ethidium bromide staining. For sequences of the primers used, see Supplementary Table 2. The MDM2 SNP309 locus (rs2279744) was genotyped in the peripheral white blood cell DNA of 70 of the 73 astrocytic glioma patients in the series, using previously published primers and standard PCR conditions (Bond et al, 2004). The $T$ to $G$ variation at the 309th nucleotide of intron 1 of the MDM2 gene was sequenced using an ABI PRISM 3100-Avant Genetic Analyser (Applied Biosystems, Warrington, UK) and Accelrys Gene 2.0 (Accelrys, Cambridge, UK) sequencing analysis software.

\section{RT - PCR of TP53 and P1- and P2-MDM2 transcripts}

Total RNA was extracted from tumour pieces and cell lines as described (Ichimura et al, 1996). The generation of cDNA and the PCR conditions for all the experiments have been previously reported (Ichimura et al, 1996, 2008; Liu et al, 2005). For sequences of the primers used, see Supplementary Table 2. Primer pairs PC180/50 and PC446/50 were used to amplify exons 4-9 and exons 7-9 of the TP53 cDNA, respectively. To amplify the P1- and P2MDM2 transcripts, forward primers PC3176 and PC3600 in the non-coding exons 1 or 2, respectively, were used with a common reverse primer PC3291 (exon 3) or PC3238 (exon 12) of the MDM2 cDNA. Standard cycling parameters (35 cycles) were used for the qualitative RT-PCR analysis on P1- and P2-MDM2 transcript expression. Products were carried out on $2 \%$ agarose gels and visualised by ethidium bromide staining. The real-time analysis of MDM2 mRNA was performed using a LightCycler ${ }^{\mathrm{R}}$ in an identical manner to that described previously for a similar analysis of other genes (Ichimura et al, 2008), but using primer pairs PC3176/ PC3291 and PC4570/PC4573 (see Supplementary Table 2) for the quantitation of P1- and P2-MDM2 transcript levels, respectively.

\section{Statistical analysis}

For the statistical analysis, the raw data obtained from the quantitative RT - PCR analysis were transformed into log2 values. To assess the effect of MDM2 gene status (amp or no amp) on P1- and P2-MDM2 mRNA levels, a Mann-Whitney $U$ test was performed using glioblastomas with $M D M 2$ amplification, wt/wt TP53 and wt/wt $p 14^{A R F}$ vs glioblastomas with no MDM2 amplification, wt/wt TP53 and wt/wt $p 14^{A R F}$. A Mann-Whitney $U$ test was also used to compare the P1- and P2-MDM2 mRNA expression within different tumour grades (GBs $v s$ AAs and As) that have no aberrations on MDM2, TP53 and $p 14^{A R F}$ genes. A two-way ANOVA was used to test the effect of TP53 and $P 14^{A R F}$ gene status or their combination on P1 and P2 transcript levels. For the latter test, tumours were separated into two categories: (i) those with wt/wt allelic status and (ii) those with at least one defective allele (i.e., $\mathrm{wt} / \mathrm{mut}$, wt/-, mut/mut, mut/- and -/-). Survival curves were obtained using the Kaplan-Meier method and statistical differences were analysed using the log-rank test. A Mann-Whitney $U$ test was used to compare the age at diagnosis for glioblastomas in relation to the SNP309 genotype. Differences were accepted as significant for $P<0.05$. All statistical analyses were performed using Minitab 13.0 and SPSS 15.0 software packages.

\section{RESULTS}

Transcription from the $\mathrm{P} 2$ promoter can be independent of p53 in astrocytic gliomas

To investigate whether expression using the P2 promoter can occur in the absence of p53, we studied P1- and P2-MDM2 mRNA expression in a TP53-null glioma cell line (Tp265MG). Multiplex PCR and RT - PCR were first used respectively to confirm the TP53 homozygous deletion and lack of endogenous TP53 mRNA in Tp265MG (Figure 1A and B). To identify the presence of P1- and P2-derived MDM2 transcripts in Tp265MG, primer sets that amplify the two distinct $5^{\prime}$ UTRs of MDM2 mRNA were used. Tp265MG was shown to express exon 2-derived transcripts (Figure 1C), and quantitative PCR confirmed the presence of P2 transcripts albeit at a very low level (Table 1).

In a preliminary study, the promoter usage of the MDM2 gene was also investigated in a series of primary astrocytic tumours, comprising of 45 GBs, 5 AAs and 5 As (Supplementary Table 1) as well as in two additional glioblastoma cell lines (CCF-STTG1 and Tp365MG) and 3 glioblastoma xenografts (GB217X4, GB181X13 and GB166X1) with known MDM2, TP53 and $p 14^{A R F}$ gene status 
Table I Gene status of MDM2, TP53 and p / 4RF, PI - and P2-MDM2 transcript levels of each specimen and SNP309 genotype and age at diagnosis of the corresponding patient

\begin{tabular}{|c|c|c|c|c|c|c|c|c|}
\hline Specimen no. & MDM2 & TP53 & $p / 4^{A R F}$ & $P I^{\mathbf{a}}$ & $\mathbf{P} \mathbf{2}^{\mathbf{a}}$ & $\mathbf{P I / P 2}$ & SNP309 & Age at diagnosis \\
\hline A54 & No amp & $w t / w t$ & $w t / w t$ & 0.68 & 2.02 & 0.337 & $\mathrm{~T} / \mathrm{T}$ & NA \\
\hline A25 & No amp & $w t / w t$ & $w t / w t$ & 0.42 & 0.52 & 0.808 & $T / T$ & NA \\
\hline A7 & No amp & $w t / w t$ & $w t / w t$ & 0.07 & 0.49 & 0.143 & $\mathrm{G} / \mathrm{T}$ & NA \\
\hline A23 & No amp & $w t / w t$ & $w t / w t$ & 0.58 & 0.63 & 0.921 & $T / T$ & NA \\
\hline A30 & No amp & $w t / w t$ & $w t / w t$ & 0.30 & 0.24 & 1.250 & $T / T$ & NA \\
\hline AA76 & No amp & $w t / w t$ & $w t / w t$ & 0.80 & 3.42 & 0.234 & $\mathrm{G} / \mathrm{T}$ & NA \\
\hline AA 107 & No amp & $w t / w t$ & $w t / w t$ & 0.33 & 1.89 & 0.175 & $\mathrm{G} / \mathrm{T}$ & NA \\
\hline AAIIO & No amp & $w t / w t$ & $w t / w t$ & 6.44 & 14.07 & 0.458 & $\mathrm{G} / \mathrm{T}$ & NA \\
\hline AAI5 & No amp & $w t / w t$ & $w t / w t$ & 0.36 & 0.73 & 0.493 & $\mathrm{~T} / \mathrm{T}$ & NA \\
\hline AA50 & No amp & $w t / w t$ & $w t / w t$ & 0.36 & 0.44 & 0.818 & $T / T$ & NA \\
\hline AA49 & No amp & $w t / w t$ & $-1-$ & NA & NA & NA & NA & NA \\
\hline GB246 & Amp & $w t / w t$ & $w t / w t$ & 18.28 & 9.4 & 1.945 & $\mathrm{G} / \mathrm{T}$ & 62 \\
\hline GB35 & Amp & $w t / w t$ & $w t / w t$ & 41.60 & 9.52 & 4.370 & $\mathrm{G} / \mathrm{T}$ & 71 \\
\hline GB37 & Amp & $w t / w t$ & $w t / w t$ & 50.68 & 13.86 & 3.657 & $\mathrm{~T} / \mathrm{T}$ & 40 \\
\hline GB90 & Amp & $w t / w t$ & $w t / w t$ & 65.46 & 11.44 & 5.722 & $T / T$ & 61 \\
\hline GBI 40 & Amp & $w t / w t$ & $w t /-$ & 9.91 & 5.66 & $1.75 \mid$ & $\mathrm{G} / \mathrm{T}$ & 42 \\
\hline GB223 & Amp & $w t / w t$ & $w t /-$ & 4.02 & 8.26 & 0.487 & $\mathrm{~T} / \mathrm{T}$ & 65 \\
\hline GB7 & Amp & $w t / w t$ & $w t /-$ & 129.97 & 12.06 & 10.777 & $\mathrm{~T} / \mathrm{T}$ & 41 \\
\hline GB8। & Amp & $w t / w t$ & $w t /-$ & 23.76 & 12.95 & 1.835 & $T / T$ & 65 \\
\hline GB237 & Amp & $w t /-$ & $w t / w t$ & NA & NA & NA & $\mathrm{G} / \mathrm{T}$ & 73 \\
\hline GB96 & No amp & $w t / w t$ & $w t / w t$ & 0.42 & 2.04 & 0.206 & G/G & 51 \\
\hline GB75 & No amp & $w t / w t$ & $w t / w t$ & 0.77 & 0.69 & 1.116 & $\mathrm{~T} / \mathrm{T}$ & 31 \\
\hline GBI 49 & No amp & $w t / w t$ & $w t / w t$ & 0.74 & 1.84 & 0.402 & $\mathrm{G} / \mathrm{T}$ & 61 \\
\hline GB247 & No amp & $w t / w t$ & $w t / w t$ & 0.64 & 1.85 & 0.346 & $\mathrm{G} / \mathrm{T}$ & 34 \\
\hline GB250 & No amp & $w t / w t$ & $w t / w t$ & 0.83 & 2.64 & 0.314 & $T / T$ & 12 \\
\hline GB57 & No amp & $w t / w t$ & $-1-$ & 1.16 & 2.13 & 0.545 & $\mathrm{G} / \mathrm{T}$ & 55 \\
\hline GB63 & No amp & $w t / w t$ & $-1-$ & 0.75 & 1.83 & 0.410 & $\mathrm{G} / \mathrm{G}$ & 74 \\
\hline GB8 & No amp & $w t / w t$ & $-1-$ & 0.67 & 1.76 & 0.381 & $\mathrm{G} / \mathrm{T}$ & 63 \\
\hline GB84 & No amp & $w t / w t$ & $-1-$ & 0.57 & 2.77 & 0.206 & $\mathrm{G} / \mathrm{T}$ & 72 \\
\hline GB94 & No amp & $w t / w t$ & $-1-$ & 0.91 & 4.78 & 0.190 & $\mathrm{G} / \mathrm{T}$ & 50 \\
\hline GB4I & No amp & $w t / w t$ & $-1-$ & 0.28 & 0.75 & 0.373 & $\mathrm{G} / \mathrm{T}$ & 70 \\
\hline GB9 & No amp & $w t / w t$ & $-1-$ & 1.19 & 2.47 & 0.482 & $\mathrm{~T} / \mathrm{T}$ & 68 \\
\hline GBI & No amp & $w t / w t$ & $-1-$ & NA & NA & NA & NA & NA \\
\hline GB5। & No amp & $w t /-$ & $-1-$ & NA & NA & NA & $\mathrm{T} / \mathrm{T}$ & 70 \\
\hline GB46 & No amp & $\mathrm{wt} / \mathrm{mut}$ & $w t / w t$ & 0.07 & 0.36 & 0.194 & $\mathrm{~T} / \mathrm{T}$ & 46 \\
\hline GB59 & No amp & wt/mut & $w t / w t$ & 1.14 & 5.03 & 0.227 & $T / T$ & 51 \\
\hline GB22I & No amp & wt/mut & $w t / w t$ & 0.10 & 0.48 & 0.208 & $\mathrm{G} / \mathrm{T}$ & 48 \\
\hline GB193 & No amp & wt/mut & $w t /-$ & 0.37 & 1.43 & 0.259 & $\mathrm{G} / \mathrm{T}$ & 56 \\
\hline GB27 & No amp & mut/mut & $w t / w t$ & 1.61 & 0.47 & 3.426 & $\mathrm{~T} / \mathrm{T}$ & 31 \\
\hline GBI64 & No amp & mut/- & $w t / w t$ & 0.29 & 1.96 & 0.148 & $\mathrm{G} / \mathrm{T}$ & 63 \\
\hline GB6I & No amp & mut/- & $w t / w t$ & 3.00 & 1.45 & 2.069 & $\mathrm{~T} / \mathrm{T}$ & 68 \\
\hline GBI03 & No amp & mut/- & $w t / w t$ & 4.84 & 1.32 & 3.667 & $\mathrm{G} / \mathrm{T}$ & 72 \\
\hline GBI3I & No amp & mut/- & $w t / w t$ & 1.37 & 3.63 & 0.377 & $\mathrm{~T} / \mathrm{T}$ & 68 \\
\hline GB29 & No amp & mut/- & $w t /-$ & 0.09 & 0.93 & 0.097 & $\mathrm{~T} / \mathrm{T}$ & 45 \\
\hline GBI 32 & No amp & mut/- & $w t /-$ & 1.51 & 2.5 & 0.604 & $\mathrm{G} / \mathrm{T}$ & 52 \\
\hline GBI7 & No amp & $\mathrm{wt} / \mathrm{mut}$ & $-1-$ & 0.86 & 0.61 & 1.410 & $\mathrm{G} / \mathrm{T}$ & 62 \\
\hline GBI38 & No amp & $\mathrm{wt} / \mathrm{mut}$ & $-1-$ & 0.22 & 0.36 & 0.611 & $\mathrm{G} / \mathrm{G}$ & 55 \\
\hline GB22 & No amp & $\mathrm{wt} / \mathrm{mut}$ & $-1-$ & 0.31 & 0.71 & 0.437 & $\mathrm{~T} / \mathrm{T}$ & 43 \\
\hline GB5 & No amp & $\mathrm{wt} / \mathrm{mut}$ & $-1-$ & 1.07 & 1.29 & 0.829 & $T / T$ & 56 \\
\hline GB4 & No amp & wt/mut & $-1-$ & NA & NA & NA & NA & NA \\
\hline GBI66 & No amp & mut/- & $-1-$ & 0.31 & 0.5 & 0.620 & $\mathrm{~T} / \mathrm{T}$ & 68 \\
\hline
\end{tabular}


Table I (Continued)

\begin{tabular}{|c|c|c|c|c|c|c|c|c|}
\hline Specimen no. & MDM2 & TP53 & $p / 4^{A R F}$ & $\mathbf{P} \mathbf{I}^{\mathbf{a}}$ & $\mathbf{P} \mathbf{2}^{\mathbf{a}}$ & $\mathbf{P} \mid / \mathbf{P} \mathbf{2}$ & SNP309 & Age at diagnosis \\
\hline GBI 6 & No amp & mut/- & $-1-$ & 0.14 & 0.12 & 1.167 & $\mathrm{G} / \mathrm{T}$ & 73 \\
\hline GB33 & No amp & mut/- & $-1-$ & 0.52 & 0.41 & 1.268 & $\mathrm{G} / \mathrm{G}$ & 66 \\
\hline GB55 & No amp & mut/- & $-1-$ & 1.42 & 2.01 & 0.706 & $\mathrm{~T} / \mathrm{T}$ & 74 \\
\hline GB2 $17 \times 4^{b}$ & Amp & $w t / w t / w t$ & $w t / w t$ & 28.8 & 2.58 & 11.162 & NA & NA \\
\hline$G B \mid 81 \times 13^{b}$ & No amp & $w t / w t$ & $-1-$ & NA & NA & NA & $\mathrm{T} / \mathrm{T}$ & NA \\
\hline GBI66X1 ${ }^{b}$ & No amp & mut/- & $-1-$ & 0.03 & 0.44 & 0.068 & NA & NA \\
\hline CCF-STTGI ${ }^{C}$ & Amp & $w t / w t$ & $w t /-$ & NA & NA & NA & NA & NA \\
\hline Tp365MG ${ }^{c}$ & Amp & $w t / w t$ & $w t /-$ & NA & NA & NA & NA & NA \\
\hline Tp265MG ${ }^{c}$ & No amp & $-1-$ & $-1-$ & 0.41 & 0.01 & 41 & $G / G$ & NA \\
\hline
\end{tabular}

$\mathrm{A}=$ astrocytoma; $\mathrm{AA}=$ anaplastic astrocytoma; $\mathrm{GB}=$ glioblastoma; no amp=no amplification; amp = amplification ( $\geqslant 5$ copies); wt/wt=two wild-type copies; $-/-=$ homozygous deletion (see text); wt/ $-=$ loss of one allele; wt/mut = retention of one wild-type allele and one mutated allele; mut/mut = both alleles with unique mutations;

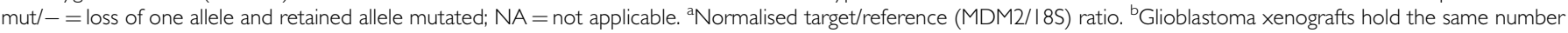
as the tumour from which they were derived with the suffix $X$ followed by passage number. ' $G$ lioblastoma cell lines.

using promoter-specific primers. Both $\mathrm{P} 1$ - and P2-derived transcripts were detectable in all the samples including the tumours and xenografts that lack wild-type TP53 (Supplementary Figure 1 and data not shown).

The majority of the primary tumours (67 of 73), the xenografts GB217X4 and GB166X1 and the cell line Tp265MG were examined by real-time PCR analysis confirming the P1- and P2-MDM2 usage (Table 1 and Supplementary Table 1). The P1/P2 ratio varied markedly from specimen to specimen, as might be expected due to the different known status of components of the $\mathrm{p} 53$ pathway and the content of normal cells in tumour specimens. The P1/P2 ratio was maximum in the Tp265MG cell line (Table 1). While it might be argued that in the tumour tissue samples analysed, all P2derived transcripts originated from the normal cells (i.e., endothelial cells, pericytes, macrophages and so on) present, the P2 transcripts from the Tp256MG line and the GB166X1 xenograft (no wild-type TP53 and use of human specific primers) could only have originated from the tumour cells. In addition, it is interesting that the transcripts in AA90 (homozygous deletion of TP53) show a $\mathrm{P} 1 / \mathrm{P} 2$ ratio of 0.47 . Overall, these findings provide evidence that p53 is not necessary for low-level P2-MDM2 transcription in astrocytic glioma cells.

\section{Variation in the usage of the $\mathrm{P} 1$ and $\mathrm{P} 2$ promoters in astrocytic gliomas}

Tumours with $M D M 2$ amplification were grouped and compared to those with no MDM2 amplification. This comparison showed a statistically significant increase in mRNA levels from both the P1 promoter $(P=0.004$, Mann - Whitney $U$ test, Figure $2 \mathrm{~A})$ and the P2 promoter $(P=0.004$, Mann-Whitney $U$ test, Figure $2 \mathrm{~B})$ in the MDM2-amplified group. This indicates that both promoters are utilised in cases with amplification of the MDM2 gene. We next compared changes in the P1/P2 ratio in the same groups (Table 1). There was a significant difference in the $\mathrm{P} 1 / \mathrm{P} 2$ ratio between tumours with $M D M 2$ amplification as compared to those without amplification $(P=0.01$, Mann - Whitney $U$ test, Figure $2 \mathrm{C})$, indicating that the elevated MDM2 mRNA expression seen in the MDM2-amplified astrocytic tumours is not equally driven by both promoters. MDM2 amplification had a higher impact on P1 than P2 expression, as indicated from the comparison of the medians of (a) P1 expression in the amplified cases (median $=22) v s$ P1 in the non-amplified cases (median $=0.74$ ) and (b) $\mathrm{P} 2$ expression in the amplified cases (median $=9.46) v s \mathrm{P} 2$ in the non-amplified cases (median $=1.85)$.

The effect of TP53 and $p 14^{A R F}$ gene status was then investigated to evaluate whether any TP53 mutation (wt/mut, mut/mut or mut/-), $p 14^{A R F}$ hemi- and nullizygosity (wt/- or -/-) or a combination of the two have an effect on P1 and/or P2 transcript levels. A two-way ANOVA indicated that any TP53 mutation was significantly associated with lower P2-MDM2 mRNA levels $(P=0.014$, two-way ANOVA, Figure 2D) and did not affect the activity of the $\mathrm{P} 1$ promoter $(P=0.575$, two-way ANOVA). This was also observed when changes in the P1/P2 ratio were examined with a correlation seen between TP53 gene status and the P1/P2 ratio $\left(P=0.039\right.$, two-way ANOVA, Figure 2E). Neither the $p 14^{A R F}$ gene status alone nor the combination of TP53 and $p 14^{A R F}$ aberrations had any significant correlation with P1 or P2 transcript expression, nor with $\mathrm{P} 1 / \mathrm{P} 2$ ratio $(P>0.05$, two-way ANOVA).

Finally, we tested whether there is a difference in the P1-, P2- or $\mathrm{P} 1 / \mathrm{P} 2$ ratio of MDM2 mRNA among high-grade (GBs) vs lowergrade gliomas (AAs and As) with no MDM2 gene amplification and wild-type TP53 and $p 14^{A R F}$ genes. No statistical difference was observed $(P>0.05$, Mann-Whitney $U$ test $)$, suggesting that tumour grade does not affect MDM2 mRNA expression in a TP53 and P14 ${ }^{A R F}$ wild-type background.

\section{The P2 promoter polymorphism (SNP309) does not appear} to be significant in astrocytic gliomas

To document the incidence of the SNP309 (rs2279744) polymorphism in astrocytic gliomas, DNA from peripheral white blood cells of 70 primary cases was studied (Supplementary Table 1). In addition to these, a glioblastoma cell line (Tp265MG) and a glioblastoma xenograft (GB181X13) were also investigated.

Table 1 summarises the genotypes obtained. Of the 70 patients genotyped for the SNP309, 34 (48.5\%) were T/T, 27 (38.5\%) were $\mathrm{G} / \mathrm{T}$ and $9(12.8 \%)$ were G/G. These frequencies are very similar to those seen in healthy Caucasian volunteers $(48 \% \mathrm{~T} / \mathrm{T}, 40 \% \mathrm{~T} / \mathrm{G}$ and $12 \% \mathrm{G} / \mathrm{G}$ ) (Bond et al, 2004). Notably, TP53-null specimens, Tp265MG (see above) and AA90 (confirmed by array-CGH, manuscript in preparation) were homozygous for the variant $G$ allele.

To examine the effect of SNP309 on MDM2 expression, we compared the levels of P2-MDM2 mRNA in glioblastomas without MDM2 gene amplification in relation to SNP309 genotype (Figure 2F). The results showed that glioblastomas homozygous for G/G did not have significantly higher levels of P2-MDM2 mRNA expression when compared to glioblastomas homozygous for $\mathrm{T} / \mathrm{T}$ at this locus. Overall, the expression levels between the different SNP309 genotypes (T/T, G/T and G/G) did not appear to be substantially affected by the polymorphism at this locus.

As shown by others (Bond et al, 2004; Swinney et al, 2005) the age of cancer formation in patients with SNP309 (G/G) can vary greatly from those individuals with a T/T genotype. We compared the age distribution at the time of diagnosis for 54 glioblastoma patients who had homozygous T/T genotype with that of patients who had either heterozygous (G/T) or homozygous (G/G) variant 
genotypes at SNP309 (Table 1). The median age at diagnosis was identical for $T / T$ genotype and $G / T$ or $G / G$ genotype (61 years). There was no association between $\mathrm{T}$ and $\mathrm{G}$ carriers in relation to age at diagnosis in glioblastomas $(P=0.658$, Mann - Whitney $U$ test).

A Kaplan-Meier analysis was performed to test if the occurrence of this gene alteration had an effect on the prognosis of the glioblastoma patients. Patients without the polymorphism $(\mathrm{T} / \mathrm{T})$ showed a median survival of 337 days, whereas patients carrying a $\mathrm{G}$ allele (G/T or G/G) had a median survival of 193 days. Even though the median survival values appear quite different, only a borderline statistical association $(P=0.052)$ in the KaplanMeier curves from glioblastomas with T/T compared with a G/G or

A
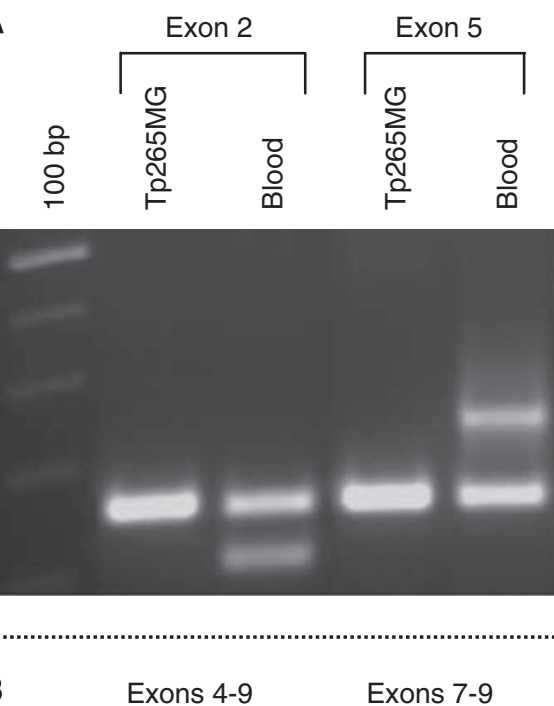

Exons 4-9

B
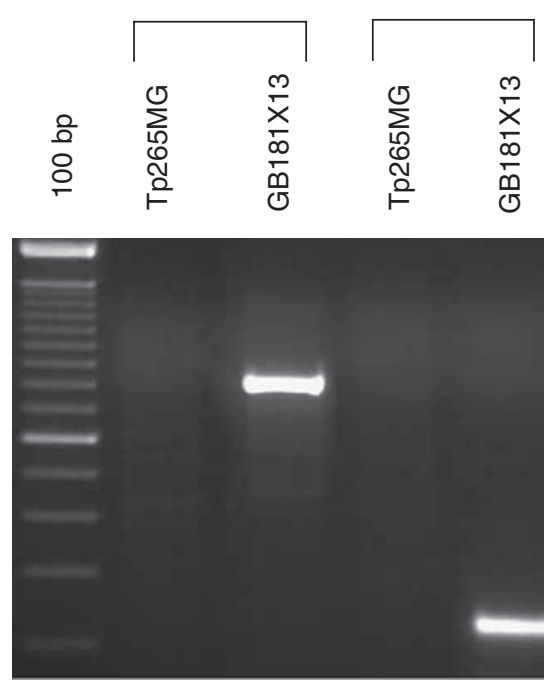

TP53

(818 bp)

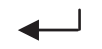

TP53

(226 bp)

C P1-MDM2

P2-MDM2

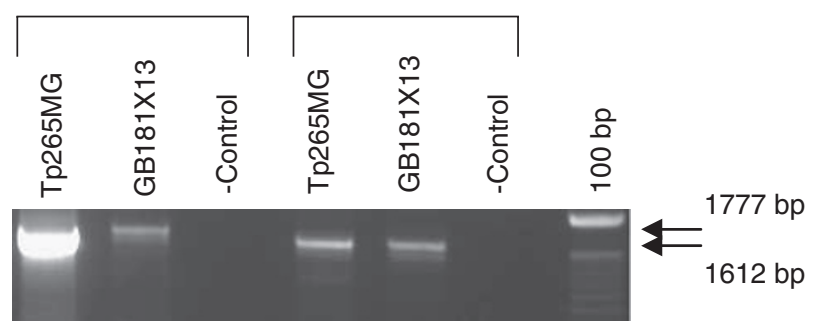

G/T genotype could be shown in the relatively small group $(n=54)$ (Figure $3 \mathrm{~A}$ ). In addition, the effect on survival of $\mathrm{G} / \mathrm{G}$ (as compared to $\mathrm{G} / \mathrm{G}+\mathrm{G} / \mathrm{T}$ ) against $\mathrm{T} / \mathrm{T}$ in glioblastoma patients was also tested and no significant association was observed $(P=0.341$, log-rank test). Although it has been suggested that the oestrogen signalling pathway allows the $G$ allele to accelerate tumour formation in women (Bond and Levine, 2007), while our data did not include any $\mathrm{G} / \mathrm{G}$ females, a preliminary comparison of the G/T against T/T female patients with glioblastoma did not show any association with survival ( $P=0.748$, log-rank test).

A prognostic value of TP53 gene status in relation to SNP309 genotype has not been demonstrated in glioblastomas. Therefore, we investigated whether SNP309 status might interact with differing TP53 status to modify the survival of glioblastoma patients. Of the 54 glioblastomas analysed, $35(65 \%)$ had wild-type TP53 (wt/wt or wt/-) and 19 (35\%) had at least one mutated TP53 allele (wt/mut, mut/- or mut/mut). As shown by Kaplan-Meier analyses, SNP309 status did not affect the association between TP53 gene status and survival in glioblastomas (Figure 3B).

We also examined the impact of $p 14^{A R F}$ gene status, as it is one of the central nodes in the p53 pathway. In the glioblastoma series studied, 22 of $54(41 \%)$ had wild-type $p 14^{A R F}$ alleles, whereas 32 of $54(59 \%)$ had lost at least one wild-type $p 14^{A R F}$ allele. As shown in Figure 3C, SNP309 status did not act synergistically with $p 14^{A R F}$ gene status to significantly alter survival in glioblastomas.

\section{DISCUSSION}

The usage of the two promoters of the MDM2 gene was analysed here for the first time in astrocytic tumours, glioblastoma xenografts and glioblastoma cell lines with known MDM2, TP53 and $p 14^{A R F}$ gene status. By documenting P1- and P2-MDM2induced transcripts in a glioblastoma cell line (Tp265MG) with no MDM2 gene amplification and homozygously deleted TP53 and p14 ${ }^{A R F}$, it was demonstrated that exon 2-derived transcripts, that is, transcripts initiated from the supposedly p53-responsive promoter (P2), were detectable even in the complete absence of all protein products of the TP53 gene. Similar findings were made in an anaplastic astrocytoma case (AA90) with no MDM2 gene amplification, homozygously deleted TP53 and two wild-type copies of $p 14^{A R F}$ (Table 1). In addition, GB103 retains only one allele of TP53 with the R175H mutation (Backlund et al, 2003) and the tumour also expresses MDM2 transcripts from the P2 promoter. The $\mathrm{R} 175 \mathrm{H}$ mutation would affect all known splice variants of TP53 (Bourdon et al, 2005) and has been shown to be transactivation defective (Soussi and Wiman, 2007).

P1- and P2-induced transcripts were expressed from other astrocytic gliomas of the series, which also lacked wild-type TP53. There were seven glioblastoma cases (GB164, GB61, GB131, GB29,

Figure I Agarose gels electrophoresis of PCR products confirming the homozygous deletion of TP53 in Tp265MG and showing that expression from the P2 promoter is independent of TP53 status. (A) Multiplex PCR using genomic DNA as template and independent pairs of primers (Supplementary Table 2) for exons 2 and 5 of TP53, and an unrelated retained locus (DEPDC5), confirming the homozygous deletion of TP53 in Tp265MG. (B) RT-PCR analysis of TP53 transcripts encompassing exons 4-9 and exons 7-9 of the TP53 CDNA showing that no TP53 mRNA was detectable in TP265MG. cDNA from the GBI8IXI3 xenograft (TP53 $3^{\mathrm{wt} / \mathrm{wt}}$ ) was used as a control template. (C) RT-PCR analysis of MDM2 transcripts using a forward primer located in either exon I (PC3 176; 5' PI promoter) or exon 2 (PC3600; 5' P2 promoter) with a common reverse primer (PC3238; sequence equivalent to $3^{\prime}$ UTR) and cDNA as template to amplify PI- and P2-MDM2 transcripts in Tp265MG. cDNA from GBI8IXI3 (TP53 ${ }^{\text {wt/wt }}$, expected to express MDM2 mRNA from both promoters) was used as a control. Note that MDM2 transcripts are expressed from both $\mathrm{PI}$ and $\mathrm{P} 2$ promoters in Tp265MG glioma cell line and in $\mathrm{GBI} 8|\mathrm{X}| 3$. 

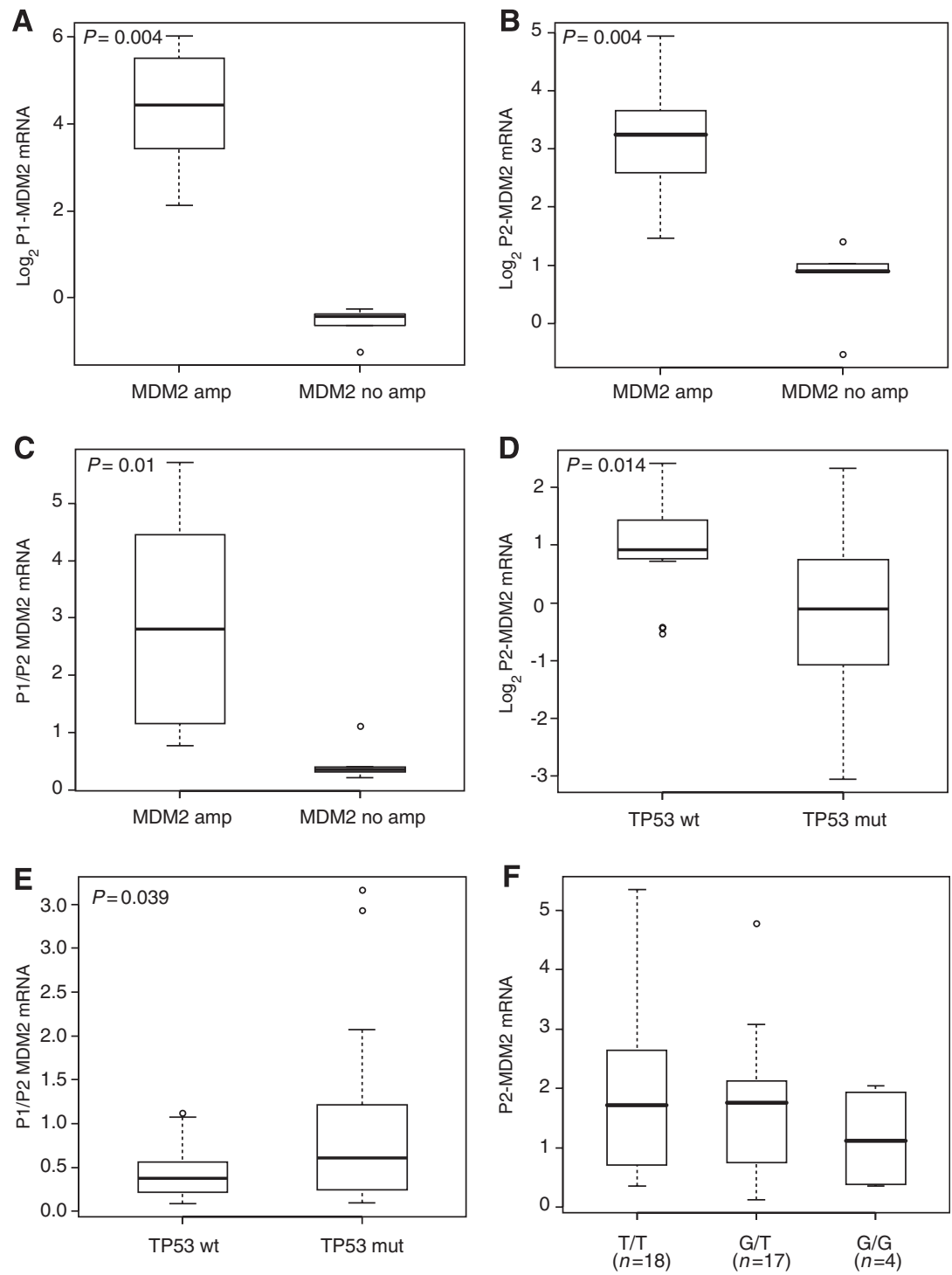

Figure 2 Box plot diagrams showing the distribution of the MDM2 mRNA levels (i.e., PI, P2 or PI/P2) obtained for glioblastomas in relation to their MDM2 gene status (amplification or no amplification), TP53 gene status (wild-type or mutation) and SNP309 genotype (T/T, G/T or G/G). The upper and lower limits of the boxes and the line across the boxes indicate the 75th, 25th percentiles and the median, respectively. The upper and lower horizontal bars indicate the 95th and 5th percentiles, respectively. Outliers are illustrated as circles. P-values are indicated at the top of the plots and were determined using the Mann-Whitney $U$ test or the two-way ANOVA (see 'Results' section for details). (A and B) Log2 PI- and Log 2 P2-MDM2 mRNA expression in glioblastomas with and without MDM2 gene amplification. (C) PI/P2 MDM2 mRNA ratio in glioblastomas with and without MDM2 gene amplification. (D) Log2 P2-MDM2 mRNA expression in glioblastomas with wild-type or mutated TP53. (E) PI/P2 MDM2 mRNA ratio in glioblastomas with wild-type or mutated TP53. (F) P2-MDM2 mRNA expression levels in glioblastomas with no MDM2 amplification in relation to their T/T, G/T or G/G genotypes for the SNP309 locus. Expression levels were measured as described in the 'Materials and Methods' section. There was no statistically significant difference in the expression levels between the $T / T, G / T$ or $G / G$ genotypes.

GB166, GB16 and GB33) that showed loss of one TP53 allele and mutation of the DNA-binding domain in the retained allele (Ichimura et al, 2000; Backlund et al, 2003). Such mutations affect all the proteins encoded by TP53 transcript variants (Bourdon et al, 2005). While there is no information about the impact on function of the specific mutations affecting each case, these seven tumours can only generate mutant p53 tetramers, which according to the model proposed by Chan et al (2004) will be inactive. Finally, GB132 retained only one mutated TP53 copy (R342X), affecting the sequence coding for the p53 oligomerisation domain, and this would prevent the formation of full-length p53 tetramers and the oligomerisation of the six other isoforms known to utilise this region of code (i.e., p $53 \beta, \mathrm{p} 53 \gamma, \Delta 40 \mathrm{p} 53 \beta, \Delta 40 \mathrm{p} 53 \gamma, \Delta 133 \mathrm{p} 53 \beta$ and $\Delta 133 \mathrm{p} 53 \gamma$ ), but would not impact on two p53 isoforms (i.e., $\Delta 40$ p53 and $\Delta 133$ p53) (Ichimura et al, 2000; Backlund et al, 2003; Bourdon et al, 2005).

While it can be argued that the P2-derived MDM2 transcripts came from normal cells present in the tumour tissue analysed, both the Tp265MG cell line and the GB166X1 xenograft contain no normal human cells, yet both expressed the P2 transcripts (primers used for analysis of the xenografts were human-sequence specific) and this would argue for at least some of the P2 transcripts coming from the tumour cells themselves. Overall, our findings provide evidence that (i) wild-type p53 is not necessary to induce MDM2 transcription through the p53-responsive promoter (P2) and that (ii) other transcriptional factor(s) can regulate MDM2 mRNA expression through P2 in astrocytic gliomas that lack endogenous p53 protein expression. 

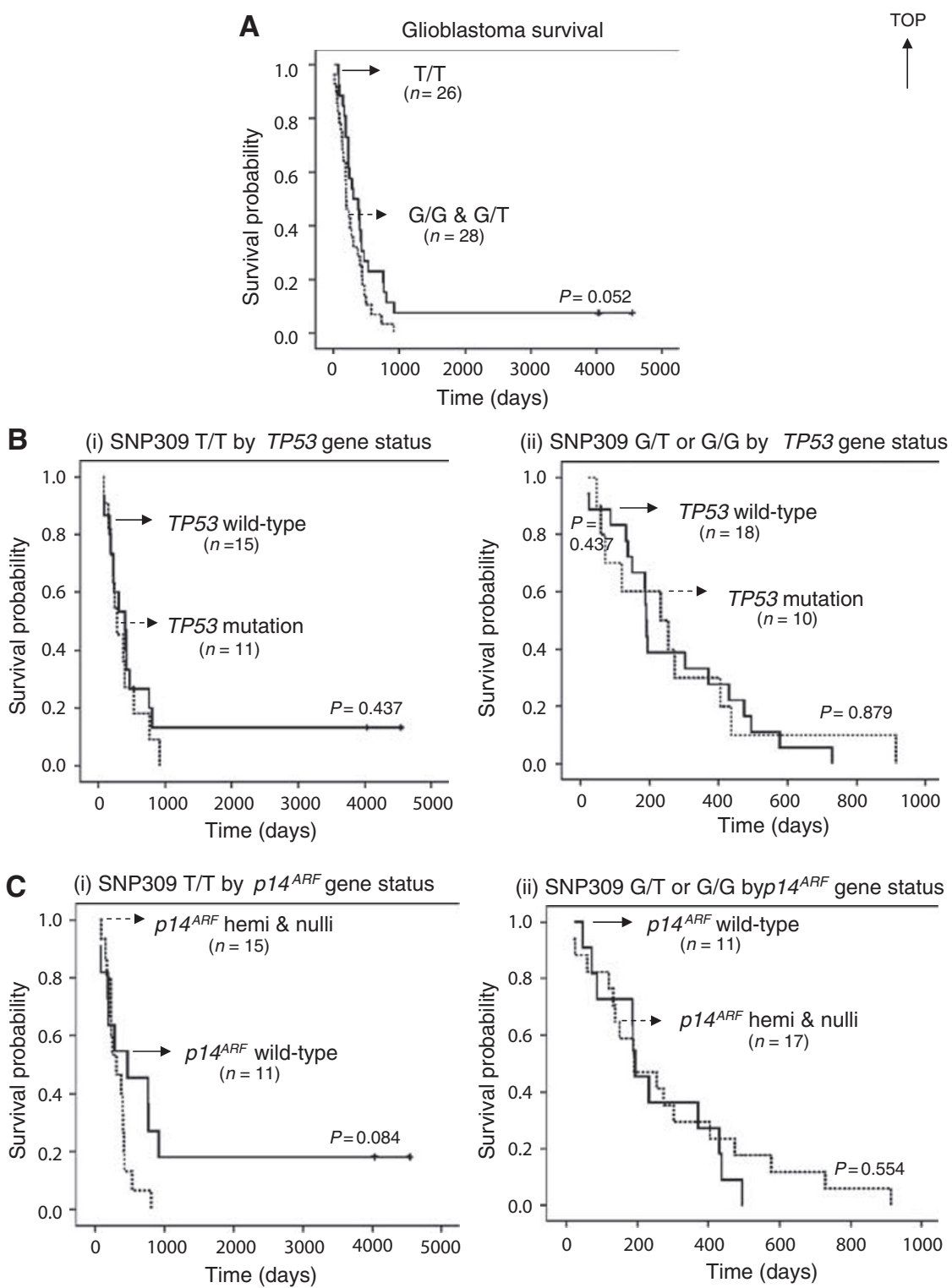

Figure 3 SNP309 in relation to glioma tumourigenesis. (A) Survival curves of patients with glioblastoma, comparing those with T/T to those with G/G or G/T for MDM2 SNP309. A borderline association in survival based on the presence or absence of the G allele was observed. (B) MDM2 SNP309 genotype in glioblastomas and TP53 status (i.e., wild-type or mutation) in relation to survival. (i) Survival curves of glioblastoma patients with the common SNP309 genotype (T/T) by TP53 gene status. (ii) Survival curves of glioblastoma patients with the variant SNP309 genotype (G/T or G/G) by TP53 gene status. (C) MDM2 SNP309 genotype of glioblastomas and p/4 ARF gene status (i.e., wild-type or hemi/nullizygosity) in relation to survival. (i) Survival curves of glioblastoma patients with the common SNP309 genotype (T/T) by p $14^{A R F}$ gene status. (ii) Survival curves of glioblastoma patients with the variant SNP309 genotype $(\mathrm{G} / \mathrm{T}$ or $\mathrm{G} / \mathrm{G})$ by $p / 4^{A R F}$ gene status. The crosses indicate censored patients. Note that no significant difference was observed in survival in conjunction with TP53 or p $14^{\text {ARF }}$ gene status.

Studies have shown that different p73 isoforms can transactivate the P2-MDM2 promoter in p53-null cells (Zhu et al, 1998; Alarcon-Vargas et al, 2000; Wang et al, 2001), although this is debated ( $\mathrm{Yu}$ et al, 1999). The TP73 gene is located at 1p36, a region reported to show deletions in astrocytic gliomas (Barbashina et al, 2005). However, this series of cases has been studied in detail as to their copy-number status at 1 p36 and this has shown that the majority of the tumours have two copies of TP73 (Ichimura et al, 2008). The 13 cases that had only one copy of TP73 (A50, A23, A30, AA34, AA49, GB96, GB250, GB3, GB52, GB56, GB63, GB84 and GB41) retained two wild-type copies of TP53 (Ichimura et al, 2008). Thus, the p53-independent expression of P2-MDM2 could be explained by $\mathrm{p} 73$ in astrocytic gliomas. Less is known about the TP63 gene, but the p63 $\alpha$ isoform has also been shown to weakly activate the MDM2 promoter in H1299, a p53-null non-small cell lung carcinoma cell line (Dohn et al, 2001).

Other functional, p53-independent elements have been reported in the $\mathrm{P} 2$ promoter. These include two thyroid hormone response elements (Qi et al, 1999) and an AP1-ETS motif together with a non-conserved upstream repeat sequence (nnGGGGC) ${ }_{5}$ (Phelps et al, 2003). At present, there are no data to indicate the relevance of p53-independent cis-acting elements for P2-MDM2 promoter activity in gliomas with no wild-type TP53.

In an attempt to provide further insights into the contribution of $\mathrm{P} 1$ and P2 promoters on the regulation of MDM2 mRNA expression in astrocytic gliomas, quantitative RT-PCR analysis was used. Amplification of the MDM2 gene in glioblastomas with wild-type TP53 and $p 14^{A R F}$ alleles was clearly reflected at the transcriptional level. Although expression from both promoters 
was increased in cases with MDM2 amplification, the amplification event had a higher impact on the P1 promoter. Assuming that the MDM2/p53 autoregulatory negative feedback loop is functional at the protein level in astrocytic tumours with amplified copies of the $M D M 2$ gene, one would expect the $\mathrm{P} 1$ promoter to give rise to high MDM2 mRNA and protein levels in these neoplasms, as all MDM2 gene coded proteins with a p53-binding domain would inhibit the transcriptional activation of the $\mathrm{P} 2$ promoter by $\mathrm{p} 53$, reducing MDM2 mRNA expression from this promoter. This would result in higher MDM2 mRNA levels from the P1 promoter, as was observed.

Importantly, there also appeared to be a clear correlation between P2-MDM2 transcript levels and TP53 gene status (Figure 2D). The statistical analyses of these data are in agreement with two independent studies in oral squamous cell carcinomas and in cell lines established from head and neck cancers or sarcomas, where P2-MDM2 transcript levels correlated with TP53 gene status (Ralhan et al, 2000; Millon et al, 2001). In contrast, very little correlation was observed between P1/P2 values and p53 mutational status in human breast cancer specimens (Okumura et al, 2002).

Overall, it would seem that complete loss of wild-type p53 is not sufficient to inhibit the $\mathrm{P} 2$ promoter in astrocytic gliomas. Thus, P2 transcripts cannot be used to predict the presence of transcriptionally active p53 in astrocytic gliomas as has been suggested in other tumours by some authors (Bull et al, 1998; Millon et al, 2001).

In an attempt to further understand control of the P2 promoter in these tumours, we examined the SNP309 (rs2279744). This polymorphism in the $\mathrm{P} 2$ promoter has been found to affect the binding of the $S p 1$ transcription factor to the promoter sequence and the G allele has been associated with increased binding of Sp1 and expression of MDM2 transcripts and protein. The G allele has also been associated with accelerated tumour formation in both hereditary and sporadic cancers in humans (Bond et al, 2004). Sequencing of the SNP309 region of the P2 promoter in the peripheral blood white cell DNA of 70 astrocytic glioma patients showed that $48 \%$ of the patients were homozygous for T/T, $38 \%$ heterozygous for $T / G$ and $12 \%$ homozygous for $\mathrm{G} / \mathrm{G}$. These frequencies were similar to the small cohort of healthy Caucasian volunteers studied by Bond et al (2004). Interestingly, both Tp265 MG and AA90 with homozygous deletion of TP53 were homozygous for the $G$ allele. The $T$ to $G$ substitution, which increases the binding affinity of the $S p 1$ transcriptional activator, might facilitate MDM2 mRNA expression from the P2 promoter in these samples. However, the P2-MDM2 expression observed in astrocytic gliomas with no wild-type TP53 cannot be solely

\section{REFERENCES}

Alarcon-Vargas D, Fuchs SY, Deb S, Ronai Z (2000) p73 transcriptional activity increases upon cooperation between its spliced forms. Oncogene 19: $831-835$

Ashcroft M, Ludwig RL, Woods DB, Copeland TD, Weber HO, MacRae EJ, Vousden KH (2002) Phosphorylation of HDM2 by Akt. Oncogene 21: 1955 - 1962

Backlund LM, Nilsson BR, Goike HM, Schmidt EE, Liu L, Ichimura K, Collins VP (2003) Short postoperative survival for glioblastoma patients with a dysfunctional Rb1 pathway in combination with no wild-type PTEN. Clin Cancer Res 9: 4151-4158

Barak Y, Gottlieb E, Juven-Gershon T, Oren M (1994) Regulation of mdm2 expression by p53: alternative promoters produce transcripts with nonidentical translation potential. Genes Dev 8: 1739-1749

Barbashina V, Salazar P, Holland EC, Rosenblum MK, Ladanyi M (2005) Allelic losses at 1 p36 and 19q13 in gliomas: correlation with histologic classification, definition of a $150-\mathrm{kb}$ minimal deleted region on $1 \mathrm{p} 36$, and evaluation of CAMTA1 as a candidate tumor suppressor gene. Clin Cancer Res 11: 1119-1128 explained by the involvement of the Sp1 trans-acting factor, as 5 of $9(55.5 \%)$ of these cases were found to be homozygous for the $\mathrm{T}$ allele (Table 1).

The G/G and G/T SNP309 in patients with glioblastoma showed a borderline association with poorer survival but no correlation with age at diagnosis or with the TP53 and $p 14^{A R F}$ status of their tumours. Our data are in agreement with three independent studies, where SNP309 was shown not to have a significant involvement in glioma tumourigenesis (El Hallani et al, 2007; Tsuiki et al, 2007; Idbaih et al, 2008). Null results have also been reported in other types of cancer in relation to this polymorphism (Campbell et al, 2006; Petenkaya et al, 2006; Pine et al, 2006; Talseth et al, 2007).

Finally, to examine the effect of the SNP309 polymorphism on MDM2 expression, the MDM2 mRNA transcriptional levels obtained by quantitative RT-PCR analysis were compared with SNP309 genetic status. In contrast to previous reports (Bond et al, 2004; Hong et al, 2005; Sanchez-Carbayo et al, 2007), the data did not show that G/G patients have a significantly higher P2-MDM2 mRNA expression levels as compared with the levels seen in astrocytic glioma patients with the T/T genotype. Overall, larger, prospective studies are needed to verify whether there is a clear involvement of this or other MDM2 polymorphism(s) in glioma tumourigenesis.

In summary, we report that both the $\mathrm{P} 1$ and $\mathrm{P} 2$ promoters are used in all genetic backgrounds, including the use of the P2 promoter in TP53-null cells, indicating a p53-independent induction of transcription from $\mathrm{P} 2$. In glioblastomas with amplification of the $M D M 2$ gene, transcripts from the P1 promoter dominate despite all such cases having two wild-type TP53 alleles. We also found no clear correlation between the SNP309 (rs2279744) locus in MDM2 and age of presentation or survival in glioblastoma patients.

\section{ACKNOWLEDGEMENTS}

We thank Professor S Tavare and Dr A Lynch for their helpful discussion and advice on statistical analysis and Dr S Kocialkowski for critical reading of the manuscript. This study was supported by funding from Cancer Research UK, the Samantha Dickson Brain Tumour Trust, the Medical Research Council and the Cambridge European Trust.

Supplementary Information accompanies the paper on British Journal of Cancer website (http://www.nature.com/bjc)
Bond GL, Hu W, Bond EE, Robins H, Lutzker SG, Arva NC, Bargonetti J, Bartel F, Taubert H, Wuerl P, Onel K, Yip L, Hwang SJ, Strong LC, Lozano G, Levine AJ (2004) A single nucleotide polymorphism in the MDM2 promoter attenuates the p53 tumor suppressor pathway and accelerates tumor formation in humans. Cell 119: $591-602$

Bond GL, Levine AJ (2007) A single nucleotide polymorphism in the p53 pathway interacts with gender, environmental stresses and tumor genetics to influence cancer in humans. Oncogene 26: 1317-1323

Bourdon JC, Fernandes K, Murray-Zmijewski F, Liu G, Diot A, Xirodimas DP, Saville MK, Lane DP (2005) p53 isoforms can regulate p53 transcriptional activity. Genes Dev 19: $2122-2137$

Broll R, Stark A, Windhovel U, Best R, Strik MW, Schimmelpenning $\mathrm{H}$, Schwandner O, Kujath P, Bruch HP, Duchrow M (1999) Expression of p53 and mdm2 mRNA and protein in colorectal carcinomas. Eur $J$ Cancer 35: $1083-1088$

Bueso-Ramos CE, Manshouri T, Haidar MA, Huh YO, Keating MJ, Albitar M (1995) Multiple patterns of MDM-2 deregulation in human leukemias: 
implications in leukemogenesis and prognosis. Leuk Lymphoma 17: $13-18$

Bull EK, Chakrabarty S, Brodsky I, Haines DS (1998) mdm2-P2 transcript levels predict the functional activity of the p53 tumor suppressor in primary leukemic cells. Oncogene 16: 2249-2257

Campbell IG, Eccles DM, Choong DY (2006) No association of the MDM2 SNP309 polymorphism with risk of breast or ovarian cancer. Cancer Lett 240: $195-197$

Chan WM, Siu WY, Lau A, Poon RY (2004) How many mutant p53 molecules are needed to inactivate a tetramer? Mol Cell Biol 24: 3536-3551

Chang CJ, Freeman DJ, Wu H (2004) PTEN regulates Mdm2 expression through the P1 promoter. J Biol Chem 279: 29841-29848

Collins VP (1983) Cultured human glial and glioma cells. Int Rev Exp Pathol 24: $135-202$

Dohn M, Zhang S, Chen X (2001) p63alpha and DeltaNp63alpha can induce cell cycle arrest and apoptosis and differentially regulate p53 target genes. Oncogene 20: 3193-3205

El Hallani S, Marie Y, Idbaih A, Rodero M, Boisselier B, Laigle-Donadey F, Ducray F, Delattre JY, Sanson M (2007) No association of MDM2 SNP309 with risk of glioblastoma and prognosis. J Neurooncol 85: $241-244$

Haupt Y, Maya R, Kazaz A, Oren M (1997) Mdm2 promotes the rapid degradation of p53. Nature 387: 296-299

Hong Y, Miao X, Zhang X, Ding F, Luo A, Guo Y, Tan W, Liu Z, Lin D (2005) The role of P53 and MDM2 polymorphisms in the risk of esophageal squamous cell carcinoma. Cancer Res 65: 9582-9587

Ichimura K, Bolin MB, Goike HM, Schmidt EE, Moshref A, Collins VP (2000) Deregulation of the $\mathrm{p} 14 \mathrm{ARF} / \mathrm{MDM} 2 / \mathrm{p} 53$ pathway is a prerequisite for human astrocytic gliomas with G1-S transition control gene abnormalities. Cancer Res 60: $417-424$

Ichimura K, Schmidt EE, Goike HM, Collins VP (1996) Human glioblastomas with no alterations of the CDKN2A (p16INK4A, MTS1) and CDK4 genes have frequent mutations of the retinoblastoma gene. Oncogene 13: $1065-1072$

Ichimura K, Vogazianou AP, Liu L, Pearson DM, Backlund LM, Plant K, Baird K, Langford CF, Gregory SG, Collins VP (2008) 1p36 is a preferential target of chromosome 1 deletions in astrocytic tumours and homozygously deleted in a subset of glioblastomas. Oncogene 27: $2097-2108$

Idbaih A, Boisselier B, Marie Y, Sanson M, El Hallani S, Criniere E, Fourtassi M, Paris S, Carpentier C, Rousseau A, Mokhtari K, Combadiere C, Laigle-Donadey F, Hoang-Xuan K, Delattre JY (2008) Influence of MDM2 SNP309 alone or in combination with the TP53 R72P polymorphism in oligodendroglial tumors. Brain Res 1198C: 16-20

Jin X, Turcott E, Englehardt S, Mize GJ, Morris DR (2003) The two upstream open reading frames of oncogene mdm2 have different translational regulatory properties. J Biol Chem 278: 25716-25721

Kamijo T, Weber JD, Zambetti G, Zindy F, Roussel MF, Sherr CJ (1998) Functional and physical interactions of the ARF tumor suppressor with p53 and Mdm2. Proc Natl Acad Sci USA 95: 8292-8297

Ko JL, Cheng YW, Chang SL, Su JM, Chen CY, Lee H (2000) MDM2 mRNA expression is a favorable prognostic factor in non-small-cell lung cancer. Int J Cancer 89: $265-270$

Kubbutat MH, Jones SN, Vousden KH (1997) Regulation of p53 stability by Mdm2. Nature 387: 299-303

Landers JE, Cassel SL, George DL (1997) Translational enhancement of mdm 2 oncogene expression in human tumor cells containing a stabilized wild-type p53 protein. Cancer Res 57: $3562-3568$

Liu L, Backlund LM, Nilsson BR, Grander D, Ichimura K, Goike HM, Collins VP (2005) Clinical significance of EGFR amplification and the aberrant EGFRvIII transcript in conventionally treated astrocytic gliomas. J Mol Med 83: $917-926$

Louis DN, Ohgaki H, Wiestler OD, Cavenee WK (2007) WHO Classification of Tumours of the Central Nervous System. IARC: Lyon

Meek DW, Knippschild U (2003) Posttranslational modification of MDM2. Mol Cancer Res 1: $1017-1026$

Mendrysa SM, Perry ME (2000) The p53 tumor suppressor protein does not regulate expression of its own inhibitor, MDM2, except under conditions of stress. Mol Cell Biol 20: 2023-2030

Millon R, Muller D, Schultz I, Salvi R, Ghnassia JP, Frebourg T, Wasylyk B, Abecassis J (2001) Loss of MDM2 expression in human head and neck squamous cell carcinomas and clinical significance. Oral Oncol 37: 620-631

Miyajima K, Tamiya S, Oda Y, Adachi T, Konomoto T, Toyoshiba H, Masuda K, Tsuneyoshi M (2001) Relative quantitation of p53 and MDM2 gene expression in leiomyosarcoma; real-time semi-quantitative reverse transcription-polymerase chain reaction. Cancer Lett 164: 177-188
Momand J, Jung D, Wilczynski S, Niland J (1998) The MDM2 gene amplification database. Nucleic Acids Res 26: 3453-3459

Momand J, Zambetti GP, Olson DC, George D, Levine AJ (1992) The mdm-2 oncogene product forms a complex with the p53 protein and inhibits p53-mediated transactivation. Cell 69: $1237-1245$

Okumura N, Saji S, Eguchi H, Nakashima S, Saji S, Hayashi S (2002) Distinct promoter usage of $\mathrm{mdm} 2$ gene in human breast cancer. Oncol Rep 9: $557-563$

Petenkaya A, Bozkurt B, Akilli-Ozturk O, Kaya HS, Gur-Dedeoglu B, Yulug IG (2006) Lack of association between the MDM2-SNP309 polymorphism and breast cancer risk. Anticancer Res 26: 4975-4977

Phelps M, Darley M, Primrose JN, Blaydes JP (2003) p53-independent activation of the hdm2-P2 promoter through multiple transcription factor response elements results in elevated hdm 2 expression in estrogen receptor alpha-positive breast cancer cells. Cancer Res 63: 2616-2623

Phelps M, Phillips A, Darley M, Blaydes JP (2005) MEK-ERK signaling controls Hdm2 oncoprotein expression by regulating hdm2 mRNA export to the cytoplasm. J Biol Chem 280: 16651-16658

Phillips A, Darley M, Blaydes JP (2006) GC-selective DNA-binding antibiotic, mithramycin $\mathrm{A}$, reveals multiple points of control in the regulation of $\mathrm{Hdm} 2$ protein synthesis. Oncogene 25: 4183-4193

Pine SR, Mechanic LE, Bowman ED, Welsh JA, Chanock SC, Shields PG, Harris CC (2006) MDM2 SNP309 and SNP354 are not associated with lung cancer risk. Cancer Epidemiol Biomarkers Prev 15: $1559-1561$

Qi JS, Yuan Y, Desai-Yajnik V, Samuels HH (1999) Regulation of the mdm2 oncogene by thyroid hormone receptor. Mol Cell Biol 19: 864-872

Ralhan R, Sandhya A, Meera M, Bohdan W, Nootan SK (2000) Induction of MDM2-P2 transcripts correlates with stabilized wild-type p53 in beteland tobacco-related human oral cancer. Am J Pathol 157: 587-596

Reifenberger G, Liu L, Ichimura K, Schmidt EE, Collins VP (1993) Amplification and overexpression of the MDM2 gene in a subset of human malignant gliomas without p53 mutations. Cancer Res 53: $2736-2739$

Ries S, Biederer C, Woods D, Shifman O, Shirasawa S, Sasazuki T, McMahon M, Oren M, McCormick F (2000) Opposing effects of Ras on p53: transcriptional activation of mdm2 and induction of p19ARF. Cell 103: $321-330$

Sanchez-Carbayo M, Socci ND, Kirchoff T, Erill N, Offit K, Bochner BH, Cordon-Cardo C (2007) A polymorphism in HDM2 (SNP309) associates with early onset in superficial tumors, TP53 mutations, and poor outcome in invasive bladder cancer. Clin Cancer Res 13: 3215-3220

Schmidt EE, Ichimura K, Goike HM, Moshref A, Liu L, Collins VP (1999) Mutational profile of the PTEN gene in primary human astrocytic tumors and cultivated xenografts. In J Neuropathol Exp Neurol 58: 1170-1183

Seng TJ, Ichimura K, Liu L, Tingby O, Pearson DM, Collins VP (2005) Complex chromosome 22 rearrangements in astrocytic tumors identified using microsatellite and chromosome 22 tile path array analysis. Genes Chromosomes Cancer 43: 181-193

Soussi T, Wiman KG (2007) Shaping genetic alterations in human cancer: the p53 mutation paradigm. Cancer Cell 12: 303-312

Swinney RM, Hsu SC, Hirschman BA, Chen TT, Tomlinson GE (2005) MDM2 promoter variation and age of diagnosis of acute lymphoblastic leukemia. Leukemia 19: 1996-1998

Talseth BA, Meldrum C, Suchy J, Kurzawski G, Lubinski J, Scott RJ (2007) MDM2 SNP309 $\mathrm{T}>\mathrm{G}$ alone or in combination with the TP53 R72P polymorphism does not appear to influence disease expression and age of diagnosis of colorectal cancer in HNPCC patients. Int J Cancer 120: $563-565$

Tsuiki H, Nishi T, Takeshima H, Yano S, Nakamura H, Makino K, Kuratsu J (2007) Single nucleotide polymorphism 309 affects murin-double-minute 2 protein expression but not glioma tumorigenesis. Neurol Med Chir (Tokyo) 47: 203-208

Wang XQ, Ongkeko WM, Lau AW, Leung KM, Poon RY (2001) A possible role of p73 on the modulation of p53 level through MDM2. Cancer Res 61: $1598-1603$

Yu J, Zhang L, Hwang PM, Rago C, Kinzler KW, Vogelstein B (1999) Identification and classification of p53-regulated genes. Proc Natl Acad Sci USA 96: 14517-14522

Zauberman A, Flusberg D, Haupt Y, Barak Y, Oren M (1995) A functional p53-responsive intronic promoter is contained within the human $\mathrm{mdm} 2$ gene. Nucleic Acids Res 23: 2584-2592

Zhang Z, Zhang R (2005) p53-independent activities of MDM2 and their relevance to cancer therapy. Curr Cancer Drug Targets 5: 9-20

Zhu J, Jiang J, Zhou W, Chen X (1998) The potential tumor suppressor p73 differentially regulates cellular p53 target genes. Cancer Res 58: 5061 - 5065 